\title{
Is Global 2D-Strain Able to Be Better Tool for Cardiotoxicity Determination in Cancer Survivors Received Various Chemotherapeutic Agents?
}

\author{
Alexander E Berezin* \\ Internal Medicine Department, State Medical University, Zaporozhye, Ukraine
}

Received: January 30, 2016; Accepted: February 24, 2016; Published: March 04, 2016

*Corresponding author: Alexander E. Berezin, Internal Medicine Department, State Medical University, 26, Mayakovsky av., Zaporozhye, Ukraine, Postcode 69035; Tel: +380612894585; E-mail:dr_berezin@mail.ru

\begin{abstract}
The cardiac toxicity has been recognized as a major medical problem that negatively affecting quality of life and prognosis in cancer survivors within chemotherapies and after ending of the treatment. The mini review is focused on the innate mechanisms of cardiotoxicity and the techniques regarding the cardiac evaluation of cancer patients. It has discussed radial and circumferential strain rates as a tool for the early detection of cardiotoxicity, whereas there are some limitation to correctly interpret findings in cancer patients with known cardiac disease and heart failure. It is concluded that more investigations are required to explain the advantages and weakness of 2D strain / 2D strain rate in cardiotoxicity determination.
\end{abstract}

Keywords: Cardiotoxicity; Anti-Neoplastic Chemotherapy; Biomarkers; Global Longitudinal Strain; Risk Stratification

\section{Abbreviations}

CREC: Cardiac Review and Evaluation Committee; HF: Heart Failure; LVEF: Left Ventricular Ejection Fraction; CTCAE: Common Terminology Criteria for Adverse Events Developed by the National Cancer Institute.

\section{Introduction}

Cardiotoxicity in cancer survivors who receive chemotherapy may correspond with premature Cardiovascular (CV) events. Modern chemotherapy agents direct against several molecular targets such as anti-epidermal growth factor receptor signaling including anti-HER2 and anti-EGFR1, anti-VEGF signaling, antimammalian target of rapamycin (mTOR), Tyrosine Kinase Inhibitors (TKIs) and Anti- Mesenchymal-Epithelial Transition (MET) [1]. To improve the survival of patients with advanced cancer, the combinations of two or more targeted agents are used. In fact, early CV toxicity may interfere with very anticancer therapies needed to enhance survivorship or arising after cancer treatment completion. As a result, the increased rate of $\mathrm{CV}$ toxicity and decreased quality of life in relation to agent' dose / location and co-morbidities is expected $[2,3]$.

It has noted that different agents, i.e. anthracyclines, anti-
HER2 agents (trastuzumab, pertuzumab), angiogenesis inhibitors (sunitinib, sorafenib, axitinib), anti-MET might distinguish in their ability to induce various CV toxicity (Figure 1). AnthracyclineBased Chemotherapy (Anth-bC) may associate with subclinical CV disease manifested by deteriorations in Left Ventricular Ejection Fraction (LVEF) or increases in aortic stiffness due to direct cell injury via DNA-damage-response pathway, suppression of protein synthesis, inducing mitochondrial dysfunction, ischemia-related necrosis and cardiac cell apoptosis [4,5]. Anti-HER2 agents appear direct toxicity via oxidative stress activation, suppression of protein synthesis, loss of myocyte population, as well as inducing cardiac hypertrophy that leads to myocardial dysfunction and heart failure presentation [6,7]. Angiogenesis inhibitors may induce hypertension, ischemia, endothelial dysfunction, and LH hypertrophy that associate with development of impaired LV diastolic function and heart failure with preserved LVEF $[8,9]$. Therefore, angiogenesis inhibitors are cable to modulate thrombosis and thromboembolism affected right ventricle pump function [10]. Several anti-MET agents are under investigation, while they exhibit $\mathrm{CV}$ toxicity due to impairment of endothelial function, worsening endothelial integrity, direct cell injury, and inducing coagulation / thrombosis [11]. Finally, target agents may realize $\mathrm{CV}$ toxicity through various mechanisms that it is essential for understanding to choose an optimal method for CV disease manifestation and progression.

Although three types of cardiotoxicities are currently recognized: acute, early-onset chronic and late-onset chronic, most cardiotoxicity occurs within the first year and is associated with agent doses and cardiac dysfunction at the end of treatment [12]. However, a prolongation of life duration following cancer treatment is greatly improved, techniques that ensure early detection and timely management of cardiotoxicity are essential [13]. Early detection and prompt therapy of cardiotoxicity appears crucial for substantial recovery of cardiac function. In this context, two-dimensional myocardial strain up to 12 months after chemotherapy might improve monitoring protocols for LV systolic function. The aim of the mini review is summary of knowledge regarding possibility of global myocardial strain to 


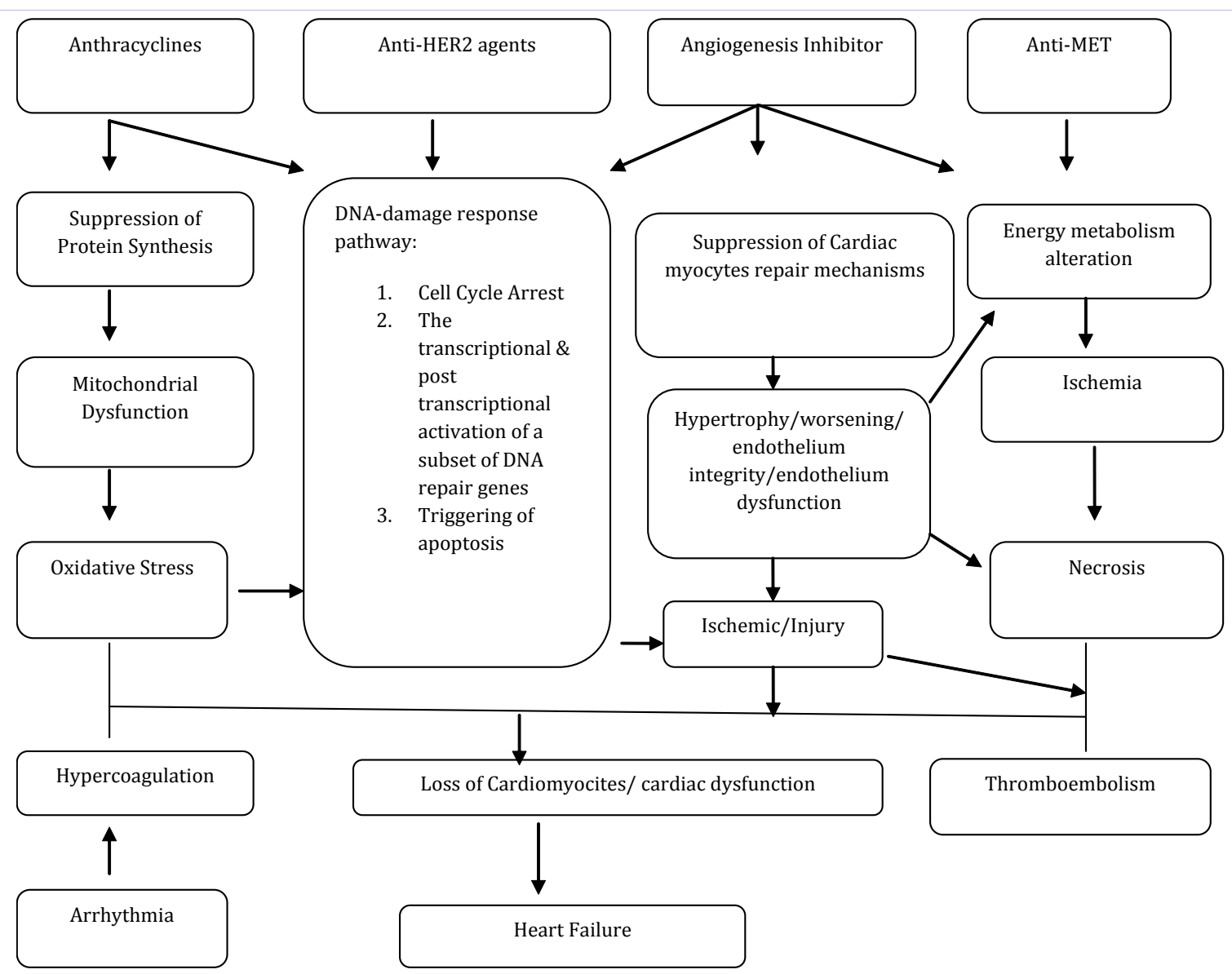

Figure 1: The capability of drugs to induce cardiotoxicity: principal mechanisms.

predict the development of cardiotoxicity in cancer survivors and advantages of the approach compared with other biomarkers use.

\section{Definition of Cardiotoxicity}

According Cardiac Review and Evaluation Committee criteria cardiotoxicity due to tumoricidal drug use is generally characterized by an asymptomatic reduction in LVEF of $\geq 10 \%$ to $<55 \%$ or, less often, as a reduction of the LVEF of $\geq 5 \%$ to $<55 \%$ with symptoms of Heart Failure (HF) [14]. The cardiac dysfunction associated with chemotherapy leads to significantly decline of LVEF and frequently associates with asymptomatic and symptomatic HF, whereas anticancer agent-induced cardiotoxicity is most often reversible upon discontinuation of treatment and initiation of standard medical care for HF $[15,16]$. There are most widely adopted criteria for adjudicating CV disease / events is the Common Terminology Criteria For Adverse Events (CTCAE) developed by the National Cancer Institute (NCI) [17]. However, differing methodologies in measurement of LVEF parameters, various magnitude of LV dysfunction, uncertain definition of potential reversibility of cardiac dysfunction, and sufficient distinguish in risk of $\mathrm{CV}$ outcomes are causes for inconsistence challenging in definition of cardiac toxicity (Table 1).
Accordingly a "multiple hit" hypothesis the integral effect of anticancer agents on cardiac function corresponds to preexisting CV risk factors, co-morbidities including CV and metabolic disease, and probably non-modified factors, i.e. genetic predisposition, sex, age (Figure 2). In this context, there are serious limitations for identification of CV toxicity especially at early stage in subjects with known CV disease affected LV pump function.

\section{Cardiac Toxicity vs. Cardiac Hypersensitivity}

Nevertheless there is confusion, in medical literature, between cardiac toxicity and cardiac hypersensitivity following anticancer therapy. The term "hypersensitivity" is widely used in the chemotherapy literature without a common definition. Cardiac hypersensitivity is defined as an unexpected reaction with signs and symptoms not consistent with known toxicity of the drug. Most reactions are co-incident with or within hours of drug administration [18]. Indeed, hypersensitivity reactions and reactions by chemical radicals that arise in drug metabolism may directly harm the heart muscle cell [19]. Cardiac adverse drug reactions result in disturbances of the heart rhythm, negative inotropic effects, direct damage to the heart muscle cell, and reduced perfusion of heart tissue. Therefore, hypersensitivity 
Table 1: The Criteria of Cardiac Toxicity.

\begin{tabular}{|c|c|c|c|}
\hline & Criteria of Cardiac Toxicity & $\begin{array}{l}\text { Confirmation of } \\
\text { Toxicity }\end{array}$ & Cardiac \\
\hline CREC & $\begin{array}{l}\text { 1. Cardiomyopathy with reduced LVEF (global or more severe in the septum region) } \\
\text { 2. Symptomatic HF } \\
\text { 3. Objective findings associated with HF (S3 gallop, tachycardia, raised jugular vein } \\
\text { pressure, etc.) } \\
\text { 4. Reduced LVEF from baseline } \geq 5 \% \text { to }<55 \% \text { with accompanying signs and } \\
\text { symptoms of HF; or declined LVEF } \geq 10 \% \text { to }<55 \% \text { without accompanying signs } \\
\text { and symptoms of HF }\end{array}$ & Any of 4 criteria & \\
\hline $\begin{array}{l}\text { LVEF criteria in clinical } \\
\text { trial and routine clinical } \\
\text { practice }\end{array}$ & $\begin{array}{l}\text { 1. Reduced LVEF from baseline }>10 \% \text { to }<55 \% \\
\text { 2. Reduced LVEF from baseline } \geq 10 \% \text { to }<50 \% \\
\text { 3. Reduced LVEF from baseline } \geq 20 \% \text { or }>15 \% \text {, but remains }>50 \% \text { in follow up } \\
\text { period } \\
\text { 4. Any LVEF decline to }<50 \%\end{array}$ & Any of 4 criteria & \\
\hline CTCAE & $\begin{array}{l}\text { 1. Declining } 10-19 \% \text { from baseline, when resting LVEF is } 40-50 \% \\
\text { 2. Declining }>20 \% \text { from baseline, when resting LVEF is } 20-39 \% \\
\text { 3. Resting LVEF }<20 \% \\
\text { 4. Newly asymptomatic or symptomatic HF or life-threatening events related to HF or } \\
\text { death }\end{array}$ & One of criterion & \\
\hline
\end{tabular}

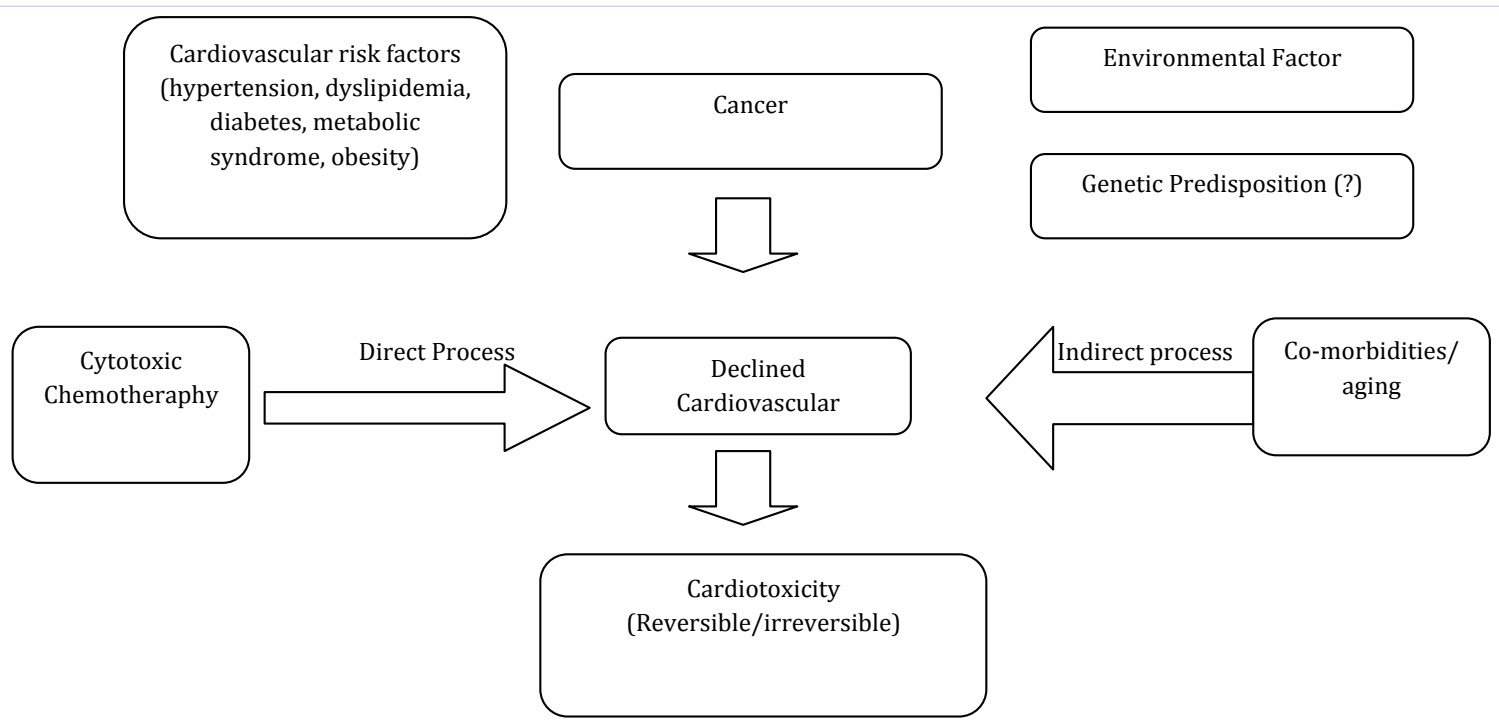

Figure 2: The "multiple hit" hypothesis elucidated the integral effect of anticancer agents on cardiac function.

is an acute not dose-dependent process that may arise at any time during treatment, while cardiotoxicity refers to chronic events and denotes dose-dependent action with progressing effects resulting in cardiac fibrosis that has never been proven in acute cardiac side effects of anticancer agents. In most cases hypersensitivity reactions are associated with the specific chemotherapeutic drug and should not mix with cardiotoxicity reactions.

\section{Modalities of Cardiac Function Evaluation}

Current methods to assess cardiac function are insensitive measures of early (subclinical) cardiac injury or / and progression of known cardiac disease. Either by 2D or 3D echocardiographies is used optionally for measurement of global and regional ejection faction. 2D-modalities supply the geometric and structural information of the myocardium with high accuracy, while 3D echocardiography permits a more detailed visualization of the anatomical proportions and may have additional value in assay of regurgitation, septal defect determination, measurement of mitral and aortic root before valve replacement therapy, and LVEF determination in subjects with previous myocardial infarction or acute coronary syndrome [20,21]. However, 2D-echocardiography may perform in several settings, whereas the acquisition of near real-time full-volume 3D data sets is influenced by the cooperation of the patient during breath holding to avoid stitching artefacts, which is limited in patients with breathless ness, arrhythmias, unstable hemodynamics, and shock.

Resting LVEF assessments, either by 2D or 3D echocardiography or nuclear blood pool scanning, as well as does not detect chemotherapy-induced early cardiomyocyte 
injury and is poor predictors of CV risk, including symptomatic $\mathrm{HF}$, particularly when LVEF is normal or mildly impaired [22-24]. In contrast, abnormal global longitudinal strain and diastolic function are more prevalent than reduced 2D and 3D LVEF and are associated with treatment exposure [25]. Thus, global longitudinal and circumferential strain might help to detect subclinical cardiac toxicity, while tissue characteristics are not able to assay with this method. However, myocardial deformation as a target of strain measurement appears to be higher availability and reproducibility for detection of early cardiac toxicity in patients with normal or near normal global LVEF without signs and symptoms of HF. Tarr et al. [26] believed that detecting myocardial dysfunction by global longitudinal and circumferential strain requires more than 3 months follow-up. Moreover, changes in rotation, twist or time-to-peak intervals could not be verified at the 3-month follow-up in the present study. Finally, 2D global radial strain seems to be the most sensitive and robust parameter to detect early myocardial damage during chemotherapy. In contrast, 3D echocardiography is not yet an established method to detect myocardial damage in clinical practice due to lower spatial and temporal resolution [27].

Toro-Salazar et al. [27] compared feasibility of echocardiographic techniques and Cardiac Magnetic Resonance Imaging (CMRI) detect subclinical cancer therapeutics-related cardiac dysfunction. Authors used declined LVEF $<55 \%$ as a criterion of cardiac toxicity in non-symptomatic HF pediatric cancer survivors with cumulative anthracycline doses $\geq 200$ $\mathrm{mg} / \mathrm{m}^{2}$. It has found that 3D echocardiographic LVEF $<55 \%$, end-systolic volume index $>29 \mathrm{~mL} / \mathrm{m}^{2}$, 3D speckle-tracking echocardiographic peak global longitudinal strain magnitude $<-17.5 \%$, and a decrease in early atrial myocardial velocity at the interventricular septum of $<10 \mathrm{~cm} / \mathrm{sec}$ by Doppler tissue imaging are the most sensitive transthoracic echocardiographic parameters to identify subjects with subclinical cardiac dysfunction. Agha et al. [28] reported that declined 2D-Specle Tracing Imaging [STE]-derived global longitudinal strain and 2D-STE-derived global longitudinal strain rate could be used for early detection of cardiotoxicity when global LVEF is near to normal. Interestingly, that STE is not only more sensitive than global LVEF evaluation in recognizing silent myocardial impairment during cancer chemotherapy, but declined global strain may be reversible and not associated with clinically significant cardiotoxicity or late development of decreased LVEF [29]. Indeed, LV dilation and subclinical impairment in cardiac function persists $>2$ years after the end of chemotherapy, without significant recovery after trastuzumab cessation, suggestive of long-term underlying cardiac damage and remodeling [30]. It is appeared to be a serious limitation to predict of $\mathrm{CV}$ mortality in long-term period and it might require novel approaches of data interpretations and methods of cardiac function examination.

However, declined LVEF defined other methods of visualizations, 2D global radial / longitudinal strain might evident once significant myocardial damage has already occurred [31]. Moreover, this magnitude of injury may be irreversible. Furthermore, peak systolic longitudinal strain rate are most useful for detection of early myocardial changes during therapy, whereas speckle tracking echocardiography and peak systolic global longitudinal strain appear to be the best measure [32]. However, CMRI has been identified a high prevalence of cardiomyopathy among adult survivors previously undiagnosed with CV disease [33]. In this context, 2D echocardiography demonstrated limited screening performance. In this high-risk population, cancer survivors with an LVEF $50 \%$ to $59 \%$ by 2D echocardiography should be considered for comprehensive cardiac assessment, which may include CMR [33].

All these findings require other methods to predict cardiac toxicity before cardiac function appearance. Probably, biomarker approach (cardiac troponins, natriuretic peptides, microRNA signatures, s100 protein, etc) might become novel method for stratification of the cancer survivors [34-36], while there is evidence regarding usefulness of biomarker strategy to predict early cardiac toxicity in declined LVEF patients receiving anthracycline-based chemotherapy [37]. In this context, serial measurements of LV systolic and diastolic function using 2D echography remain simple and routine affordable methods for determination of asymptomatic cardiac toxicity. Whether strain measurement is useful to identify non-HF patients at risk of early cardiotoxicity induced any regime of chemotherapy remains challenging [38,39]. Yet, Right Ventricular (RV) dysfunction has been persisted in the majority at follow-up, although the prognostic value of RV dysfunction at the time of cardiotoxicity warrants further investigation [40]. Some experts have suggested that involving RV in cardiomyopathy development after or within chemotherapy is required more widely using of CVRI instead strain measurement $[41,42]$. Probably, implementation of RealTime 3-D Echocardiography (RT-3DE) with CMRI in cancer survivors might help to detect more abnormalities in cardiac function than conventional measurement of global LVEF and 2D strain / 2D strain rate [41]. Moreover, it is suggested that the LV dyssynchrony indexes and tissue characteristics derived from RT-3DE and CMRI appear potentially useful in assessing the early signs of cardiotoxicity between chemotherapy and radiotherapy exposed long-term survivors of cancer.

Finally, radial and circumferential strain rates are interesting tool for the early detection of cardiotoxicity, whereas there are some limitation to correctly interpret findings in cancer patients with known cardiac disease and HF. It is required more investigations to explain the advantages and weakness of 2D strain / 2D strain rate in cardiotoxicity determination.

In conclusion, declined global strain may identify early cardiac dysfunction in non-symptomatic HF cancer survivors who may benefit from early medical intervention, when conventional echocardiographic values are normal or near normal. RT3DE alone or in combination with CMRI is more accurate for measurement of global LVEF and RV function compared with 2D- echography in subjects at higher risk for poor clinical cardiac outcomes. Contemporary 2D- echography remains probably as routine method for detecting decreased LVEF due to cardiac toxicity in survivors with asymptomatic and symptomatic HF. 


\section{References}

1. Yuan DD, Zhu ZX, Zhang X, Liu J. Targeted therapy for gastric cancer: Current status and future directions. Oncol Rep. 2016; 35(3): 124554. doi: 10.3892/or.2015.4528.

2. Drafts BC, Twomley KM, D’Agostino R Jr, Lawrence J, Avis N, Ellis LR, et al. Low to moderate dose anthracycline-based chemotherapy is associated with early noninvasive imaging evidence of subclinical cardiovascular disease. JACC Cardiovasc Imaging. 2013; 6(8): 877-85 doi: 10.1016/j.jcmg.2012.11.017.

3. Guarneri V, Broglio K, Kau SW, Cristofanilli M, Buzdar AU, Valero V, et al. Prognostic value of pathologic complete response after primary chemotherapy in relation to hormone receptor status and other factors. J Clin Oncol. 2006; 24(7): 1037-44.

4. Stoodley PW, Richards DA, Boyd A, Hui R, Harnett PR, Meikle SR, et al. Left ventricular systolic function in HER2/neu negative breast cancer patients treated with anthracycline chemotherapy: a comparative analysis of left ventricular ejection fraction and myocardial strain imaging over 12 months. Eur J Cancer. 2013; 49(16): 3396-403. doi: 10.1016/j.ejca.2013.06.046.

5. Cardinale D, Colombo A, Bacchiani G, Tedeschi I, Meroni CA, Veglia F, et al. Early detection of anthracycline cardiotoxicity and improvement with heart failure therapy. Circulation. 2016; 133(4): e363. doi: 10.1161/CIRCULATIONAHA.115.018780.

6. Moja L, Tagliabue L, Balduzzi S, Parmelli E, Pistotti V, Guarneri V, et al. Trastuzumab containing regimens for early breast cancer. Cochrane Database Syst Rev. 2012; 4: CD006243. doi: 10.1002/14651858. CD006243.pub2.

7. Lahsaee S, Corkery DP, Anthes LE, Holly A, Dellaire G. Estrogen receptor alpha (ESR1)-signaling regulates the expression of the taxane-response biomarker PRP4K. Exp Cell Res. 2016; 340(1): 12531. doi: 10.1016/j.yexcr.2015.12.013.

8. Mallick S, Gandhi AK, Rath GK. Therapeutic approach beyond conventional temozolomide for newly diagnosed glioblastoma: Review of the present evidence and future direction. Indian J Med Paediatr Oncol. 2015; 36(4): 229-37. doi: 10.4103/0971-5851.171543.

9. Qu CY, Zheng Y, Zhou M, Zhang Y, Shen F, Cao J, et al. Value of bevacizumab in treatment of colorectal cancer: A meta-analysis. World J Gastroenterol. 2015; 21(16): 5072-80. doi: 10.3748/wjg.v21. i16.5072.

10.0ppelt P, Betbadal A, Nayak L. Approach to chemotherapyassociated thrombosis. Vasc Med. 2015; 20(2): 153-61. doi: $10.1177 / 1358863 \times 14568705$.

11. Minuti G, Landi L. MET deregulation in breast cancer. Ann Transl Med. 2015; 3(13): 181. doi: 10.3978/j.issn.2305-5839.2015.06.22.

12. Mulrooney DA, Armstrong GT, Huang S, Ness KK, Ehrhardt MJ, Joshi VM, et al. Cardiac Outcomes in Adult Survivors of Childhood Cancer Exposed to Cardiotoxic Therapy: A Cross-sectional Study. Ann Intern Med. 2016; 164(2): 93-101. doi: 10.7326/M15-0424.

13. Dietz AC, Sivanandam S, Konety S, Kaufman CL, Gage RM, Kelly AS, et al. Evaluation of traditional and novel measures of cardiac function to detect anthracycline-induced cardiotoxicity in survivors of childhood cancer. J Cancer Surviv. 2014; 8(2): 183-9. doi: 10.1007/s11764-0130326-2.

14. Martin M, Esteva FJ, Alba E, Khandheria B, Perez-Isla L, Garcia-Saenz $J A$, et al. Minimizing cardiotoxicity while optimizing treatment efficacy with trastuzumab: review and expert recommendations. Oncologist. 2009; 14(1): 1-11. doi: 10.1634/theoncologist.2008-0137.
15. Tan-Chiu E, Yothers G, Romond E, Geyer CE Jr, Ewer M, Keefe D, et al. Assessment of cardiac dysfunction in a randomized trial comparing doxorubicin and cyclophosphamide followed by paclitaxel, with or without trastuzumab as adjuvant therapy in node-positive, human epidermal growth factor receptor 2-overexpressing breast cancer: NSABP B-31. J Clin Oncol. 2005; 23(31): 7811-9.

16. Ganame J, Claus P, Eyskens B, Uyttebroeck A, Renard M, D’Hooge $\mathrm{J}$, et al. Acute cardiac functional and morphological changes after Anthracycline infusions in children. Am J Cardiol. 2007; 99(7): 974-7.

17. Khouri MG, Douglas PS, Mackey JR, Martin M, Scott JM, ScherrerCrosbie M, et al. Cancer Therapy-Induced Cardiac Toxicity in Early Breast Cancer: Addressing the Unresolved Issues. Circulation. 2012; 126(23): 2749-63. doi: 10.1161/CIRCULATIONAHA.112.100560.

18. Shepherd GM. Hypersensitivity reactions to chemotherapeutic drugs. Clin Rev Allergy Immunol. 2003; 24(3): 253-62.

19. Haen E. Pharmaca Induced Cardiac Injury. Dtsch Med Wochenschr. 2016; 141(2): 103-9. doi: 10.1055/s-0041-109806.

20. Ripley B, Kelil T, Cheezum MK, Goncalves A, Di Carli MF, Rybicki FJ, et al. 3D printing based on cardiac CT assists anatomic visualization prior to transcatheter aortic valve replacement. J Cardiovasc Comput Tomogr. 2016; 10(1): 28-36. doi: 10.1016/j.jcct.2015.12.004.

21. Stoebe S, Lange K, Pfeiffer D, Hagendorff A. Feasibility of proximal right coronary artery imaging by 2D and 3D echocardiography in comparison to coronary angiography. Echo Res Pract. 2015; 2(3): 73 9. doi: 10.1530/ERP-15-0014.

22. Jurcut R, Wildiers H, Ganame J, D’Hooge J, Paridaens R, Voigt JU. Detection and monitoring of cardiotoxicity-what does modern cardiology offer? Support Care Cancer. 2008; 16(5): 437-45. doi: 10.1007/s00520-007-0397-6.

23. Watts RG, George M, Johnson WH Jr. Pretreatment and routine echocardiogram monitoring during chemotherapy for anthracyclineinduced cardiotoxicity rarely identifies significant cardiac dysfunction or alters treatment decisions: A 5-year review at a single pediatric oncology center. Cancer. 2012; 118(7): 1919-24. doi: 10.1002/ cncr.26481.

24. Ewer MS, Lenihan DJ. Left ventricular ejection fraction and cardiotoxicity: Is our ear really to the ground? J Clin Oncol. 2008; 26(8): 1201-3. doi: 10.1200/JC0.2007.14.8742.

25. Armstrong GT, Joshi VM, Ness KK, Marwick TH, Zhang N, Srivastava D, et al. Comprehensive Echocardiographic Detection of TreatmentRelated Cardiac Dysfunction in Adult Survivors of Childhood Cancer: Results From the St. Jude Lifetime Cohort Study. J Am Coll Cardiol. 2015; 65(23): 2511-22. doi: 10.1016/j.jacc.2015.04.013.

26. Tarr A, Stoebe S, Tuennemann J, Baka Z, Pfeiffer D, Varga A, et al. Early detection of cardiotoxicity by 2D and 3D deformation imaging in patients receiving chemotherapy. Echo Res Pract. 2015; 2(3): 81-8. doi: 10.1530/ERP-14-0084.

27. Toro-Salazar OH, Ferranti J, Lorenzoni R, Walling S, Mazur W Raman SV, et al. Feasibility of Echocardiographic Techniques to Detect Subclinical Cancer Therapeutics-Related Cardiac Dysfunction among High-Dose Patients When Compared with Cardiac Magnetic Resonance Imaging. J Am Soc Echocardiogr. 2016; 29(2): 119-31. doi: 10.1016/j.echo.2015.10.008.

28. Agha H, Shalaby L, Attia W, Abdelmohsen G, Aziz OA, Rahman MY. Early Ventricular Dysfunction After Anthracycline Chemotherapy in Children. Pediatr Cardiol. 2015

29. Mele D, Malagutti P, Indelli M, Ferrari L, Casadei F, Da Ros L, et al. 
Reversibility of Left Ventricle Longitudinal Strain Alterations Induced by Adjuvant Therapy in Early Breast Cancer Patients. Ultrasound Med Biol. 2016; 42(1): 125-32. doi: 10.1016/j.ultrasmedbio.2015.09.008.

30. Tan TC, Bouras S, Sawaya H, Sebag IA, Cohen V, Picard MH, et al Time Trends of Left Ventricular Ejection Fraction and Myocardial Deformation Indices in a Cohort of Women with Breast Cancer Treated with Anthracyclines, Taxanes, and Trastuzumab. J Am Soc Echocardiogr. 2015; 28(5): 509-14. doi: 10.1016/j.echo.2015.02.001.

31. Walker J, Bhullar N, Fallah-Rad N, Lytwyn M, Golian M, Fang T, et al. Role of three-dimensional echocardiography in breast cancer: Comparison with two-dimensional echocardiography, multiple-gated acquisition scans, and cardiac magnetic resonance imaging. J Clin Oncol. 2010; 28(21): 3429-36. doi: 10.1200/JC0.2009.26.7294.

32. Korzeniowska K, Jankowski J, Cieślewicz A, Jabłecka A. Current approach for detection of sub-clinical left ventricular dysfunction associated with chemotherapy. Pharmacol Rep. 2015; 67(6): 1098102. doi: 10.1016/j.pharep.2015.03.010.

33. Armstrong GT, Plana JC, Zhang N, Srivastava D, Green DM, Ness KK, et al. Screening adult survivors of childhood cancer for cardiomyopathy: Comparison of echocardiography and cardiac magnetic resonance imaging. J Clin Oncol. 2012; 30(23): 2876-84. doi: 10.1200/ JCO.2011.40.3584.

34. Cardinale D, Colombo A, Torrisi R, Sandri MT, Civelli M, Salvatici M, et al. Trastuzumab-induced cardiotoxicity: Clinical and prognostic implications of troponin i evaluation. J Clin Oncol. 2010; 28(25): 3910 6. doi: 10.1200/JC0.2009.27.3615.

35. Sandri MT, Salvatici M, Cardinale D, Zorzino L, Passerini R, Lentat $\mathrm{P}$, et al. N-terminal pro-b-type natriuretic peptide after high-dose chemotherapy: A marker predictive of cardiac dysfunction? Clin Chem. 2005; 51(8): 1405-10.

36. Cardinale D, Sandri MT. Role of biomarkers in chemotherapy- induced cardiotoxicity. Prog Cardiovasc Dis. 2010; 53(2): 121-9. doi: 10.1016/j.pcad.2010.04.002.

37. Dodos F, Halbsguth T, Erdmann E, Hoppe UC. Usefulness of myocardial performance index and biochemical markers for early detection of anthracycline-induced cardiotoxicity in adults. Clin Res Cardiol. 2008; 97(5): 318-26. doi: 10.1007/s00392-007-0633-6.

38. Jurcut R, Wildiers H, Ganame J, D’Hooge J, De Backer J, Denys H, et al. Strain rate imaging detects early cardiac effects of pegylated liposomal doxorubicin as adjuvant therapy in elderly patients with breast cancer. J Am Soc Echocardiogr. 2008; 21(12): 1283-9. doi: 10.1016/j.echo.2008.10.005.

39. Hare JL, Brown JK, Leano R, Jenkins C, Woodward N, Marwick TH. Use of myocardial deformation imaging to detect preclinical myocardial dysfunction before conventional measures in patients undergoing breast cancer treatment with trastuzumab. Am Heart J. 2009; 158(2): 294-301. doi: 10.1016/j.ahj.2009.05.031.

40. Calleja A, Poulin F, Khorolsky C, Shariat M, Bedard PL, Amir E, et al. Right Ventricular Dysfunction in Patients Experiencing Cardiotoxicity during Breast Cancer Therapy. J Oncol. 2015; 2015: 609194. doi: $10.1155 / 2015 / 609194$.

41. Ylänen K, Poutanen T, Savikurki-Heikkilä P, Rinta-Kiikka I, Eerola A, Vettenranta K. Cardiac magnetic resonance imaging in the evaluation of the late effects of anthracyclines among long-term survivors of childhood cancer. J Am Coll Cardiol. 2013; 61(14): 1539-47. doi: 10.1016/j.jacc.2013.01.019.

42. Ylänen K, Eerola A, Vettenranta K, Poutanen T. Three-dimensional echocardiography and cardiac magnetic resonance imaging in the screening of long-term survivors of childhood cancer after cardiotoxic therapy. Am J Cardiol. 2014; 113(11): 1886-92. doi: 10.1016/j. amjcard.2014.03.019. 